\title{
Effects of Parametric Speaker Sound on Physiological Functions during Mental Task
}

\author{
Soomin Lee, Tetsuo Katsuura and Yoshihiro Shimomura \\ Graduate School of Engineering, Chiba University
}

\begin{abstract}
In recent years, parametric speakers have been used in various circumstances. However, nothing has yet been demonstrated about the safety of parametric speakers for the human body. Therefore, we studied their effects on physiological functions. Nine male subjects participated in this study. They completed three consecutive sessions: a 20-min quiet period as a baseline, a 45-min mental task period with a general speaker or a parametric speaker, and a 20-min recovery period. We measured electrocardiogram (ECG), photoplethysmogram (PTG), electroencephalogram (EEG), blood pressure (BP), and baroreflex sensitivity (BRS). Two experiments, one with a general speaker (the general condition), the other with a parametric speaker (the parametric condition), were conducted at the same time of day on separate days. To examine the effects of the parametric speaker, a twoway repeated measures ANOVA (speaker factor and time factor) was conducted. We found that sympathetic nervous activity and second derivative of PTG in task period and recovery period during the parametric condition were significantly lower than those indications during the general condition. Furthermore, $\Delta$ parasympathetic nervous activity during the parametric condition in task period and recovery period tended to be smaller than that during the general condition. The results suggested that the burden of the parametric speaker is lower than that of the general speaker for physiological functions, especially those of the cardiovascular system. Furthermore, we verified that the reaction time with the parametric speaker is shorter than that with the general speaker. J Physiol Anthropol 30(1): 9-14, 2011 http://www. jstage.jst.go.jp/browse/jpa2

[DOI: $10.2114 /$ jpa2.30.9]
\end{abstract}

Keyword: parametric speaker, mental task, physiological response

\section{Introduction}

Most human beings now live in modern technological civilization which would have been absolutely unimaginable to our ancestors (Sato, 2005). In the field of physiological anthropology, we should study the human nature living in such a modern world. In modern society, the high-directional loudspeakers system has been developed (Westervelt, 1963). This high-directional loudspeakers based on a parametric array have allowed sound transmission within a narrow range of acoustic space like a 'spotlight' (Komatsuzaki et al., 2010). Several studies reported the characteristics of the parametric speaker since Westervelt (1963) had showed about the parametric speaker array (Pompei, 1999). It is well known that the sound of the parametric speaker is heard relatively well around \pm 30 degrees in the front of the speaker and that the sound of the parametric speaker is sharper than that of the general speaker. In addition, with the parametric speaker it is difficult to recognize the distance of the sound source because of the lack of reverberant sound.

Recently, the parametric speaker has been used for various situations such as an information tool in a museum and a traffic information apparatus in a station for people with visual impairments. However, nothing has yet been revealed about the safety of parametric speakers for the human body. The purpose of this study was to clarify the influence of the parametric speaker sound on the human body from the viewpoint of the physiological Anthropology.

Therefore, we measured the effects of this sound on several physiological functions and task performance and compared these with the effects of the general speaker sound.

\section{Methods}

\section{Subjects}

Nine male healthy students participated in this study. They were asked to refrain from hard exercise, drinking caffeinated beverages, and smoking cigarettes during the 2-h period immediately preceding the experiment. The subjects performed an auditory test (ITERA, GN Otometrics) before the experiment. Their hearing ability was confirmed to be normal. All subjects gave fully informed consent to participate in this study. Their physical characteristic is shown in Table 1. 


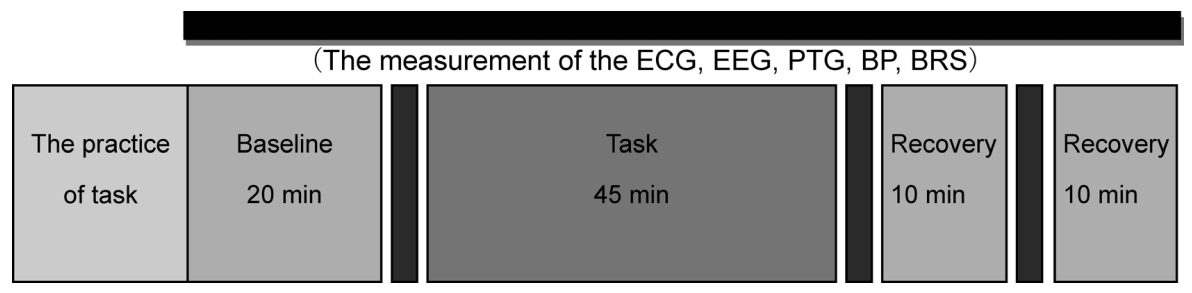

Fig. 1 The protocol of the experiments.

Table 1 Physical characteristic of the subjects

\begin{tabular}{ccccc}
\hline The subjects & Age(yr) & BMI & Height(cm) & Weight(kg) \\
\hline Sub 1 & 21 & 21.1 & 160 & 54 \\
Sub 2 & 22 & 22.3 & 164 & 60 \\
Sub 3 & 24 & 24.2 & 165 & 66 \\
Sub 4 & 23 & 18.7 & 170 & 54 \\
Sub 5 & 23 & 23.0 & 168 & 65 \\
Sub 6 & 22 & 19.3 & 172 & 57 \\
Sub 7 & 25 & 20.1 & 170 & 58 \\
Sub 8 & 25 & 20.8 & 170 & 60 \\
Sub 9 & 21 & 17.8 & 179 & 57 \\
\hline Mean \pm SD & $23 \pm 1.5$ & $20.8 \pm 2.10$ & $169 \pm 5.41$ & $59 \pm 4.3$ \\
\hline
\end{tabular}

\section{Protocol}

Two experiments were conducted at the same time of day on separate days and under the same conditions with the exception of the speaker condition (general or parametric speaker). After the subjects arrived at the acoustic room, they were asked to relax for at least $15 \mathrm{~min}$, before the recording sessions began. Subjects completed three consecutive sessions: a 20-min quiet period as a baseline, a 45 -min mental task period with a general speaker or a parametric speaker, and a 20-min recovery period. Subjects were told to rest and physically relax throughout the experimental period. The experimental protocol is shown in Fig. 1. The order of the two speaker conditions was counterbalanced between the subjects.

\section{Mental task and the sound of speaker}

The experiments were conducted in a soundproof room. The mental task consisted of both normal and deviant sentences coming from the speaker. The sound of parametric speaker was made by the following methods. Airborne ultrasound waves of $40 \mathrm{kHz}$ were dynamically Double-sideband-AM modulated with audio signals, being radiated from an ultrasound emitter. The inherent nonlinearity of the air works as a de-modulator. Thus de-modulated sounds having sharp beamwidth impinge on ear drums.

Subjects were instructed to judge whether the sentence was true or false and to push a button indicating that as quickly as possible. The sentences were made by a text-to-speech synthesis software (SMART TALK Version 3, OKI). For example, a normal sentence is "shounen wa toshokan ni itta." (a boy went to the library). In this case, the subjects were asked

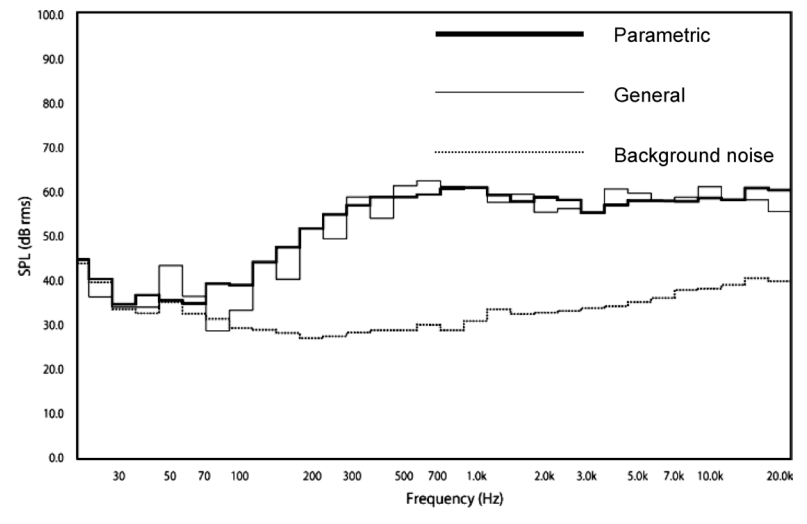

Fig. 2 The output characteristic of the general speaker and parametric speaker.

to push a "red" button. By contrast, a deviant sentence is "shounen wa ringo ni itta" (a boy went to apple). In this case, the subjects were asked to push a "blue" button. The Leq of the sound generated by the general speaker was $72.4 \mathrm{dBA}$, and the Leq of sound generated by the parametric speaker was $72.3 \mathrm{dBA}$. The background Leq of the soundproof room was $52.2 \mathrm{dBA}$. The output characteristics of the general speaker and parametric speaker were almost same, as shown in Fig. 2.

\section{Physiological parameters}

EEG activity was recorded with $\mathrm{Ag} / \mathrm{AgCl}$ electrodes affixed with electrode paste on the $\mathrm{Pz}, \mathrm{CZ}, \mathrm{Fz}, \mathrm{O} 1$, and $\mathrm{O} 2$ electrode sites of the international 10-20 system. Linked earlobes were used as a reference with a forehead ground. A bipolar electrooculogram (EOG) was recorded with electrodes placed above and below the left eye. The EEG and EOG were amplified by appropriate devices (EEG100B and EOG100B, BIOPAC Systems). EEGs were fast Fourier transformed for each $5.12 \mathrm{~s}$ of data, not including artifacts such as ocular movement. We then obtained the relative power density of the alpha wave $(8-13 \mathrm{~Hz} / 4-30 \mathrm{~Hz})$ (Yoto et al., 2007).

Continuous measurements of beat-to-beat BP were obtained with an ambulatory noninvasive BP monitor (TNO, Portapres). Beat-to-beat systolic BP and systolic-to-systolic pulse intervals were analyzed for baroreceptor reflex sensitivity (BRS) using the method of sequential analysis (Parati et al., 1989; Hughson et al., 1993; Gizdulich et al., 1996). An electrocardiogram (ECG) was recorded using an amplifier (ECG 100B, BIOPAC Systems), and digitized to derive heart rate variability (HRV) 
Table 2 Results of HR, BP, BRS, EEG alpha- band ratio

\begin{tabular}{|c|c|c|c|c|c|c|c|}
\hline & & \multicolumn{2}{|c|}{ Speaker condition } & \multicolumn{4}{|c|}{ Time periods } \\
\hline & & \multirow{2}{*}{$\begin{array}{l}\text { General } \\
\text { Speaker }\end{array}$} & \multirow{2}{*}{$\begin{array}{c}\text { Parametric } \\
\text { Speaker }\end{array}$} & \multicolumn{2}{|c|}{ General speaker } & \multicolumn{2}{|c|}{ Parametric speaker } \\
\hline & & & & Task & Recovery & Task & Recovery \\
\hline & HR & $0.87 \pm 0.43$ & $-0.75 \pm 0.40$ & $0.99 \pm 1.02$ & $0.57 \pm 1.25$ & $-0.47 \pm 0.83$ & $-1.45 \pm 1.21$ \\
\hline & SBP & $5.46 \pm 1.02$ & $4.79 \pm 0.80$ & $3.98 \pm 2.00$ & $9.15 \pm 2.93 *$ & $2.72 \pm 0.53$ & $9.97 \pm 2.36^{*}$ \\
\hline & DBP & $4.90 \pm 0.70$ & $2.94 \pm 0.45$ & $3.67 \pm 1.12$ & $8.21 \pm 2.06^{*}$ & $1.86 \pm 0.52$ & $5.65 \pm 1.28 *$ \\
\hline & BRS & $-0.09 \pm 0.43$ & $-0.95 \pm 0.41$ & $0.07 \pm 0.84$ & $-0.49 \pm 0.81$ & $-0.60 \pm 0.67$ & $-1.87 \pm 1.05$ \\
\hline \multirow{5}{*}{$\begin{array}{l}\text { EEG } \\
\text { Alpha } \\
\text { band } \\
\text { ratio }\end{array}$} & $\lceil\mathrm{Fz}$ & $0.01 \pm 0.02$ & $-0.08 \pm 0.08$ & $-0.09 \pm 0.08$ & $-0.07 \pm 0.08$ & $0.01 \pm 0.01$ & $0.03 \pm 0.02$ \\
\hline & $\mathrm{Cz}$ & $0.01 \pm 0.02$ & $0.01 \pm 0.06$ & $0.01 \pm 0.06$ & $0.01 \pm 0.07$ & $0.01 \pm 0.03$ & $0.02 \pm 0.02$ \\
\hline & $\mathrm{Pz}$ & $0.04 \pm 0.02$ & $0.03 \pm 0.08$ & $0.03 \pm 0.08$ & $0.03 \pm 0.08$ & $0.04 \pm 0.03$ & $0.05 \pm 0.03$ \\
\hline & $\mathrm{O} 1$ & $0.03 \pm 0.01$ & $0.01 \pm 0.07$ & $0.02 \pm 0.07$ & $0.02 \pm 0.07$ & $0.03 \pm 0.02$ & $0.05 \pm 0.02$ \\
\hline & L 02 & $0.05 \pm 0.01$ & $0.03 \pm 0.07$ & $0.02 \pm 0.07$ & $0.03 \pm 0.07$ & $0.04 \pm 0.02$ & $0.06 \pm 0.02$ \\
\hline
\end{tabular}

*p<0.05: it shows significant difference between Task and Recovery periods.
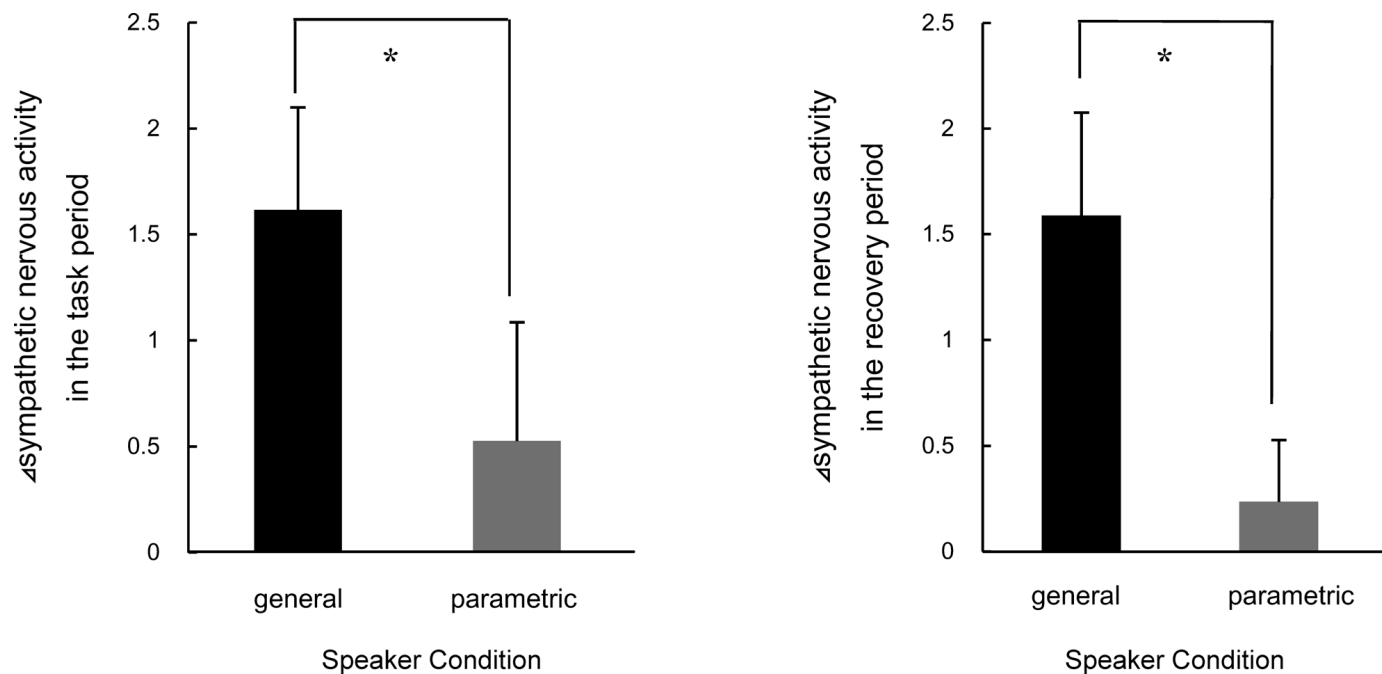

Fig. 3 the changes $(\Delta)$ in sympathetic nervous activity in the general and parametric speaker condition (means \pm S.E., ${ }^{*}: p<0.05$ ).

and heart rate (HR) using signal processing software (Mathcad, PTC). The high frequency (HF) component and low frequency (LF) component were integrated at $0.05-0.15 \mathrm{~Hz}$ and $0.15-$ $0.40 \mathrm{~Hz}$ of the power spectra, respectively (Pomeranz et al., 1985; Pagani et al., 1986). Sympathetic nervous activity (LF/ $\mathrm{HF})$ and parasympathetic nervous activity $(\mathrm{HF} /(\mathrm{LF}+\mathrm{HF}))$ were calculated. Photoplethysmogram (PTG) was recorded using an amplifier (PPG 100B, BIOPAC Systems), a low-pass filter was set at $3.0 \mathrm{~Hz}$, and a high-pass filter was set at $0.5 \mathrm{~Hz}$. We measured the $b / a$ ratio of the second derivative of PTG (SDPTG), which was considered to reflect the distensibility of the vascular wall (Takazawa et al., 1993, 1998; Otsuka et al., 2006).

All signals of the physiological indexes were converted from analog to digital at a $1 \mathrm{kHz}$ sampling rate (MP150, BIOPAC Systems) and were stored in a computer.

\section{Statistical analysis}

We calculated the mean of every $5 \mathrm{~min}$ epoch for all indexes. In the physiological parameters, changes $(\Delta)$ calculated by subtracting the respective baseline values from the average values for the task period and recovery period were used to conduct statistical analyses. A two-way repeated measures ANOVA (speaker factor $\times$ time factor) was conducted. By contrast, in the task performance, data for comparing the general speaker with the parametric speaker were tested using the paired $t$-test. When a significant $\mathrm{F}$ value was found, we performed a Student-Newman-Keuls as a post hoc test. All statistical analyses were performed using SPSS 11.0J (SPSS, Japan). Differences with values of $p<0.05$ were considered significant. Data are shown as mean \pm standard error of the mean unless otherwise stated.

\section{Results}

\section{Physiological data}

Table 2 showed that the HR, BRS and the relative power density of the alpha wave at $\mathrm{Pz}, \mathrm{Cz}, \mathrm{Fz}, \mathrm{O} 1, \mathrm{O} 2$ were not significantly different between the speaker condition and time. However, the SBP and DSP of the two-way repeated measures 

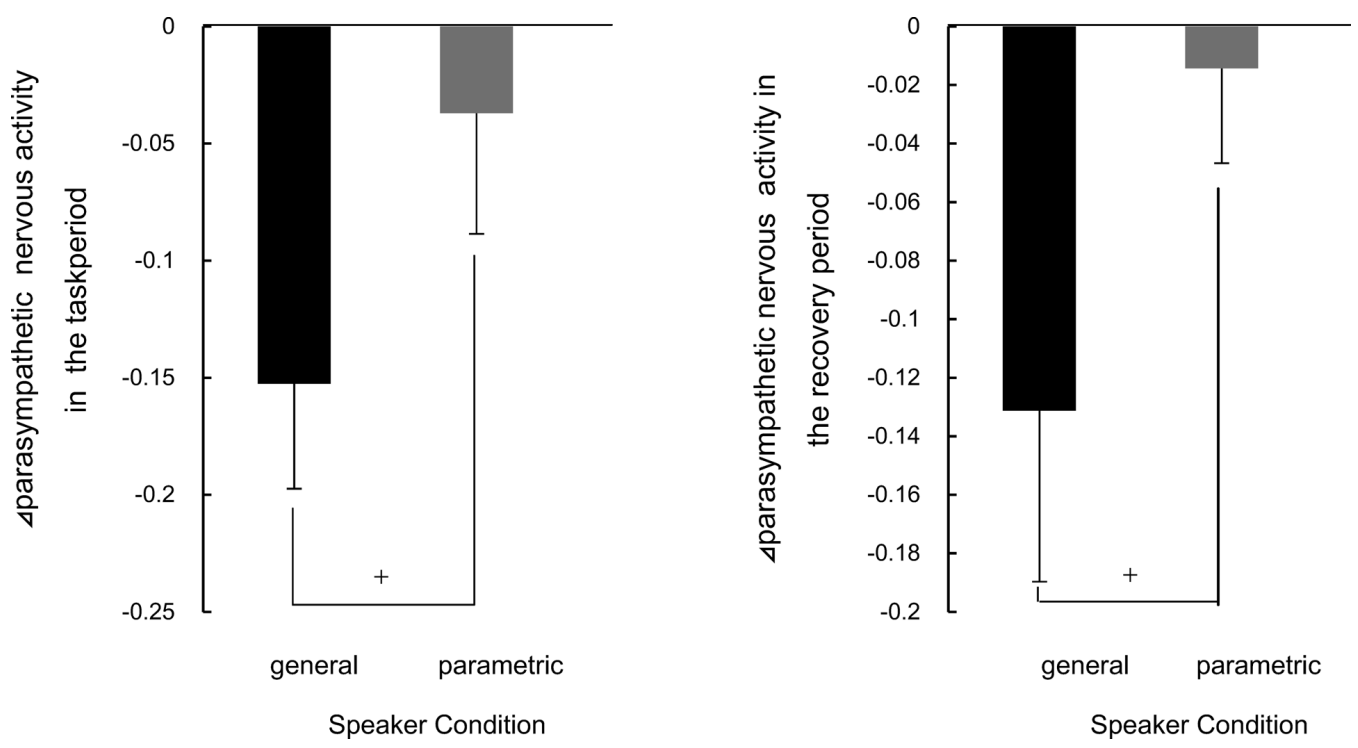

Fig. 4 The changes $(\Delta)$ in parasympathetic nervous activity in the general and parametric speaker condition (means \pm S.E., $+: p<0.1)$.
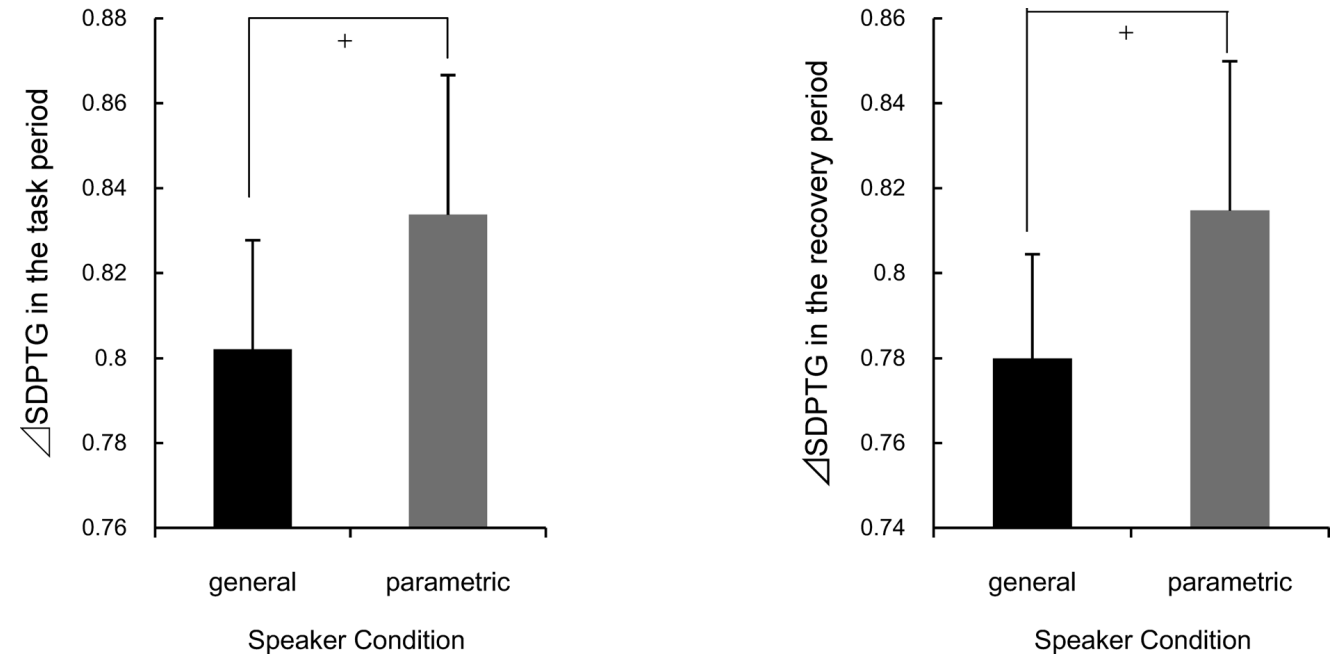

Fig. 5 The changes $(\Delta)$ in b/a ratio of second derivative of PTG (SDPTG) in the general and parametric speaker condition (means \pm S.E., + : $p<0.1$ ).

ANOVA showed that the main effects were significant for the time factor but not significant for the speaker factor.

Figure 3 shows the results of the changes $(\Delta)$ in sympathetic nervous activity. It showed that the main effects were significant for speaker factor and time factor. $\Delta$ Sympathetic nervous activity in the parametric condition was significantly lower than that in the general condition during the task period and recovery period $[\mathrm{F}(1,8)=10.024, p<0.05]$. Figure 4 shows the results of the changes $(\Delta)$ in parasympathetic nervous activity. It showed that $\Delta$ parasympathetic nervous activity in the parametric condition tended to be significantly smaller than that in the general condition during the task period and recovery period $[\mathrm{F}(1,8)=6.092, p<0.1]$. Figure 5 shows the results of the changes $(\Delta)$ of SDPTG. It showed that $\Delta$ SDPTG in the parametric condition tended to be significantly higher than that in the general speaker during the task period and recovery period $[\mathrm{F}(1,8)=4.245, p<0.1]$. The repeated measures ANOVA revealed no significant interactions of the two factors in all physiological data.

\section{Task performance}

The reaction time in the parametric condition was shorter than that in the general condition for both normal sentences $[t=2.691, p<0.05]$ and deviant sentences $[t=2.975, p<0.05]$ (Fig. 6). However, the correct rate was not significantly different between the general speaker and parametric speaker conditions for both normal sentences and deviant sentences (Fig. 7). 


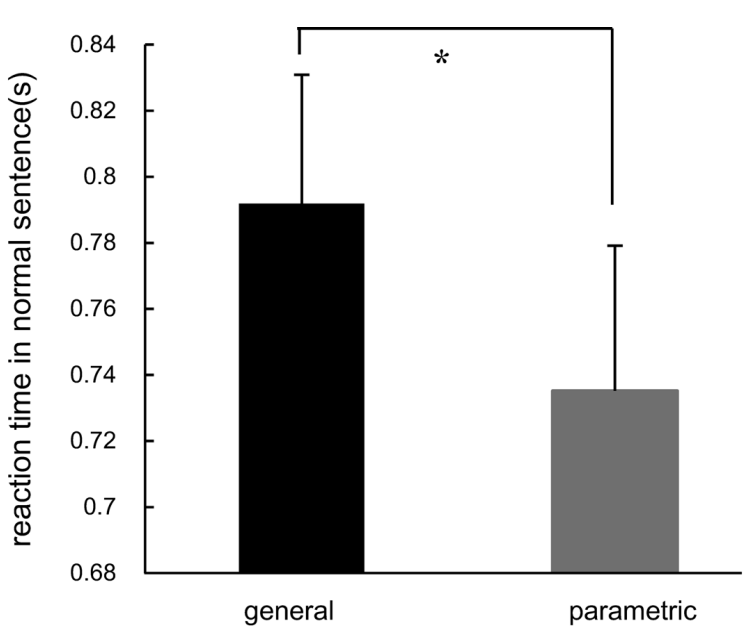

Speaker Condition

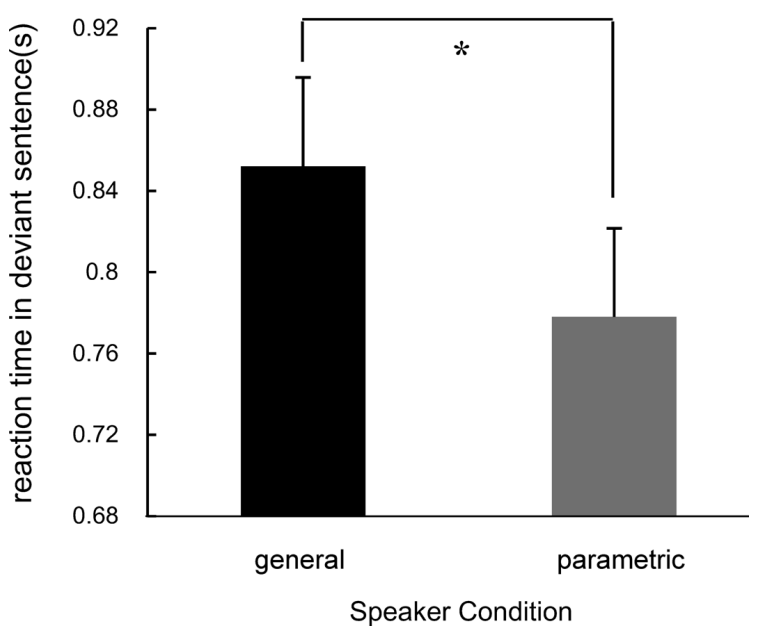

Fig. 6 The reaction time in the general and parametric speaker condition (means \pm S.E., $*: p<0.05$ ).

\section{Discussion}

The present study compared the physiological responses during the task and the recovery period between the general speaker and parametric speaker. We found that the burden during the task period and recovery period in the parametric condition was significantly lower than that in the general condition.

With regard to $\Delta$ sympathetic nervous activity in the task and recovery periods, the parametric condition was lower than that in the general condition. Furthermore, $\Delta$ parasympathetic nervous activity during the parametric condition in the task and recovery periods tended to be smaller than that during the general condition. These results suggest that the sound of the parametric speaker was less stressful than that of the general speaker with regard to physiological functions, especially those of the cardiovascular system.

On the other hand, HR showed no significant difference between the general speaker and the parametric speaker

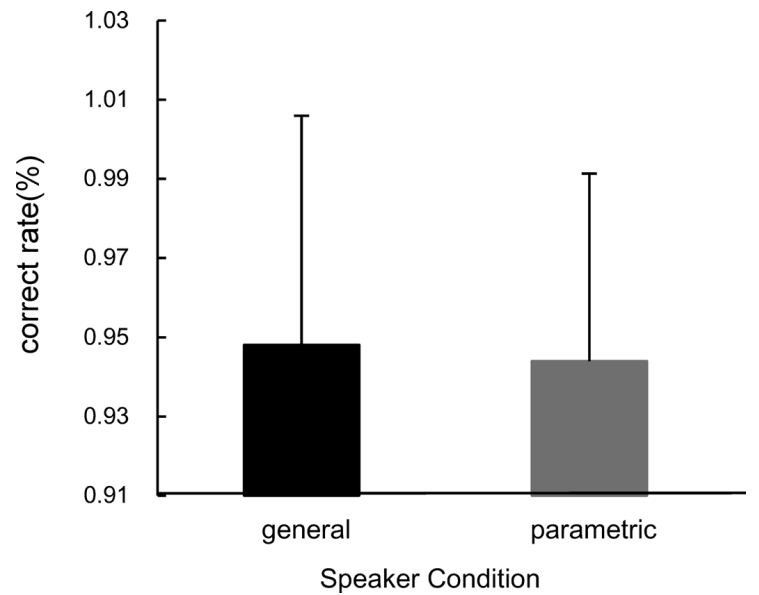

Fig. 7 The correct in the general and parametric speaker condition.

conditions. In addition, the amplitude ratio (b/a) of $\Delta$ SDPTG during the parametric condition in the task and recovery periods tended to be higher than that during the general condition. It was shown that the effect of the parametric speaker on SDPTG coincided with responses in the cardiovascular system. We believe that the subjects experienced relaxation as a result of the parametric speaker reducing the burden on the human body.

It is verified that sympathetic nervous activity and SDPTG, which reflects the sympathetic nervous system, during parametric speaker condition were significantly lower than those during the general speaker condition, but the BRS, which reflects the parasympathetic nervous system, showed no significant difference between the speaker conditions. From these results, we considered that different response pathways were a possible cause of these different results for the same stress ( Lee et al., 2009).

On the other hand, the reaction time in the task with the parametric speaker was shorter than that with the general speaker for both normal sentences and deviant sentences. We estimated that this was related to the directional characteristic of the sound of parametric speaker. There is no report on articulation and reverberation of the parametric speaker until now. However, it is well known that a characteristic of the parametric speaker is sound transmission within a narrow range of acoustic space like a 'spotlight' (Komatsuzaki et al., 2010). Croft and Norris (2001) reported that parametric speaker has a shaper directivity. In addition, with the sound of the parametric speaker it is difficult to recognize the distance of the sound source because of the lack of reverberation from the surrounding wall surfaces.

From this point of view, the parametric speaker sound might be comparable with a study in the anechoic room where there is little reverberation from the wall surfaces.

In a previous study, it was reported that the distance cognition of the sound source was difficult in the anechoic room (Nielsen et al., 1993). According to Ono (1989), it was found that a sound was clearly audible in subjects' ears in the 
anechoic room.

Meanwhile, Nabelek et al. (1989) showed that the articulation of the sound was decreased by means of reverberation. Therefore, we estimated that the reaction time of the task with the parametric speaker was shorter because of an improvement in articulation by means of the lack of reverberation from the wall surface.

In conclusion, this is the first research that shows parametric speaker sound has less stress level than general speaker sound with regard to physiological functions, especially those of the cardiovascular system. Furthermore, we found that the reaction time in the task with the parametric speaker was shorter than that with the general speaker. These results were obtained from the viewpoint of physiological anthropology which is most important in future studies to consider application of the parametric speaker sound.

It remains necessary to conduct further experiments on the effect of the parametric speaker on physiological responses based on changes in distance and frequencies of the ultrasonic wave.

Acknowledgments We are grateful to our colleagues who participated in the experiments. This study was supported in part by a grant from Panasonic Electronic Devices Co., Ltd. Especially, we thanks to Konno F, and Takeda K of Panasonic Electronic Devices for their kind cooperation.

\section{References}

Croft J, Norris J (2001) Theory, history and the advancement of parametric loudspeaker. White Paper of American Technology Corporation.

Gizdulich P, Imholz BP, van den Meiracker AH, Parati G, Wesseling KH (1996) Finapres tracking of systolic pressure and baroreflex sensitivity improved by wave form filtering. $\mathrm{J}$ Hypertens 14: 243-250

Hughson RL, Quintin L, Annat G, Yamamoto Y, Gharib C (1993) Spontaneous baroreflex by sequence and power spectral methods in humans. Clin Physiol 13: 663-676

Komatsuzaki T, Iwata Y (2010) Active noise control using high-directional parametric loudspeaker: Numerical study on radiated and interfered Filed (Mechanincal systems). Trans Jpn Soc Mechanical Engineers 76(761): 177-184 [In Japanese]

Lee S, Katsuura T, Shimomura Y, Iwanag K, Sugiura K (2009) The effect of eating a meal physiological responses during performance of a mental task. Jpn J Physiol Anthropol 14: 123-131 [In Japanese with English Abstract]

Nabelek A, Letowski T, Tucker F (1989) Reverberant overlap and self masking in consonant identification. J Acoust Soc Am 86: 1259-1265

Nielsen S (1993) Auditory distance perception in different rooms. J Audio Eng Soc 41: 755-770

Ono Y (1989) A study on comparison between an anechoic room and a field experiment in Japanese three syllable articulation test. Architectural institute of Japan. Summaries of Technical Papers of Annual Meeting Architectural Institute of Japan. D Environmental Engineering: 369-370 [In Japanese]

Ostuka T, Kawada T, Katsumata M (2006) Utility of second derivative of the finger Photoplethysmogram for the Estimation of the risk of Coronary Heart Disease in the general population. Circ J 70: 304-310

Pagani M, Lombardi F, Guzzetti S, Rimoldi O, Furlan R, Pizzinelli P, Sandrone G, Cerutti S, Malliani A (1986) Power spectral analysis of heart rate and arterial pressure variabilities as a marker of sympatho-vagal interaction in man and conscious dog. Circ Res 59: 178-193

Parati G, Casadei R, Groppelli A, Di Rienzo M, Mancia G (1989) Comparison of finger and intra-arterial blood pressure monitoring at rest and during laboratory testing. Hypertension 13: 647-655

Pomeranz B, Macaulay RJB, Caudill MA, Kutz I, Adam D, Gordon D, Kilborn KM, Barger AC, Shannon DC, Cohen RJ, Benson H (1985) Assessment of autonomic function in humans by heart rate spectral analysis. Am J Physiol 248: H151-H153

Pompei F (1999) The use of airborn ultrasonics for generating audible sound beams. J Audio Eng Soc 47: 726-731

Sato M (2005) The development of conceptual framework in physiological anthropology. J Physiol Anthropol Appl Human Sci 24: 289-295

Takazawa K, Fujita M, Yabe K (1993) Clinical usefulness of the second derivative of a plethysmogram (acceleration plethysmogram). J Cardiol 23: 207-217

Takazawa K, Tanaka N, Fujita M (1998) Assessment of vasoactive agents and vascular aging by the second derivative of photoplethysmogram waveform. Hypertension 32: 365-370

Westervelt P (1963) Parametric acoustic array. J Acoust Soc Am 35: 535-537

Yoto A, Katsuura T, Iwanaga K, Shimomura Y (2007) Effects of object color stimuli on human brain activities in perception and attention referred to EEG alpha band response. J Physiol Anthropol 26: 373-379

Received: June 10, 2010

Accepted: November 7, 2010

Correspondence to: Soomin Lee, Graduate School of Engineering, Chiba University, 1-33, Yayoi, Inage, Chiba 2638522, Japan

Phone: +81-43-290-3086

Fax: +81-43-290-3087

e-mail: yisoomin@restaff.chiba-u.jp,soominyi@gmail.com 